\title{
Effect of Hole Doping in Kagome System $\mathrm{YCO}_{5}$
}

Nileema Sharma ${ }^{1,2}$, Santosh K. ${ }^{3}$ and Madhav Prasad Ghimire ${ }^{1,2 *}$

\footnotetext{
${ }^{1}$ Central Department of Physics Tribhuvan University, Kirtipur, Kathmandu

${ }^{2}$ Condensed Matter Physics Research Center, Butwal, Rupandehi

${ }^{3}$ San José University, San Jose, United States

* madhav.ghimire@cdp.tu.edu.np
} 
With the presence of robust flat band in $\mathrm{YCO}_{5}$, which has high Magnetocrystalline Anisotropy Energy (MAE) among itinerant magnets, doping of hole with smaller ionic radii to the $\mathrm{Y}$-site has shown significant change in the MAE. This system is found to be pseudo two dimensional ferromagnetic in nature under density functional calculations employing $G G A+U$ exchange potential in WIEN2k. With hole doping the original flat band is extended to whole Brillouin zone. In addition to it the Fermi level is shifted because of it. This enables to control the filling of flat bands upon doping, resulting in novel feature of band engineering.

Keywords: Kagome magnet; Magnetocrystalline Anisotropy Energy; Density Functional Theory; Exchange interaction; Flat band 


\section{Introduction}

- Magnets

- Energy generation, storage

- Green Energy unprecedented growth in demand

- Ever increasing demand and constrained cost

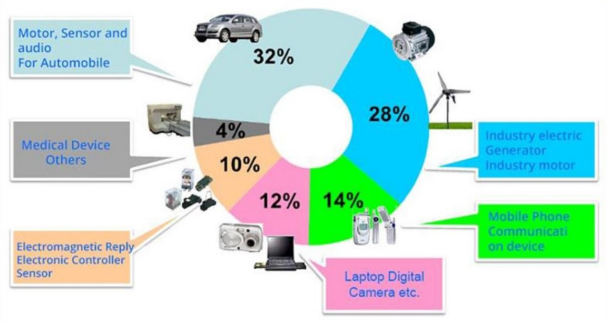

Fig: Uses of Permanent magnets (www.tcd.ie/Physics/research/groups/magnetism)

- The choice of materials is limited to include magnetic elements only

- Rare-earth based magnets including $\mathrm{Nd}_{2} \mathrm{Fe}_{14} \mathrm{~B}$ and intermetallic magnet $\mathrm{SmCO}_{5} \rightarrow$ champion hard magnets.

- YCO5 mishmetal highest anisotripy energy and Curie temperature required for the permanent magnets among itinerant magnets ${ }^{1}$.

${ }^{1}$ K. Ohashi, Nippon Kinzoku Gakkai-Shi 76 (2012) 
- Rare-earth free magnets

- Low cost and more cost efficient than rare-earth magnets

- Tunable magnetization direction in absence of rare-earth ${ }^{2}$

- High magnetization and Curie temperature becaue of transition metals

- Ytterium based magnets

- High anisotropy and susceptibility is less effected by temperature $^{3}$

- Anti-parallel coupling of $\mathrm{Y}$ - $d$ electrons with $d$ electrons of transition metals ${ }^{4}$

- Doping on Y-site to enhance the MAE without changing the contribution from Co atoms

- Tunable magnetization direction

${ }^{2}$ M. Matsumoto, R. Banerjee, and J. B. Staunton Phys. Rev. B 90 (2014)

${ }^{3}$ B. Szpunar, Physica B+C 130 (1985)

${ }^{4}$ K. Strnat, G. Hoffer, J. Olson, W. Ostertag, and J. J. Becker, Journal of Applied Physics 38 (1967) 


\section{Methodology}

- Electronic and magnetic structure calculations are done by employing Density Functional Theory (DFT).

- WIEN2k based on Full Potential Linearized Augmented Plane Wave (FP-LAPW) is used as the tool for DFT ${ }^{5}$

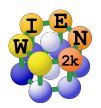

- Standard Generalized Gradient Approximation (GGA) was employed as exchange functional

- Supercell approach was used for the fractional doping on Y-site

${ }^{5}$ P. Blaha, K. Schwarz, G. K. H. Madsen, D. Kvasnicka, and J. Luitz, Technische Universität Wien, Vienna, Austria, (2001) 


\section{Structure of $\mathrm{YCO}_{5}$}

- The kagome system $\mathrm{YCO}_{5}$ belongs to hexagonal $\mathrm{CaCu}_{5}$ structure, with three inequivalent sites for $\mathrm{Y}(1 \mathrm{a}), \mathrm{Co}(2 \mathrm{c})$ and $\mathrm{Co}(3 \mathrm{~g})$.

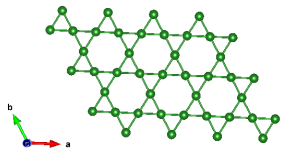

Fig: Kagome arrangement $\mathrm{Co}(3 \mathrm{~g})$

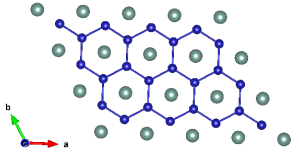

Fig: Hexagonal arrangement $\mathrm{Co}(2 \mathrm{c})$

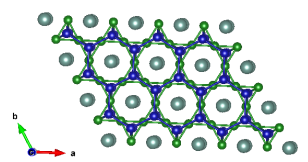

Fig: Planer structure $\mathrm{YCO}_{5}$

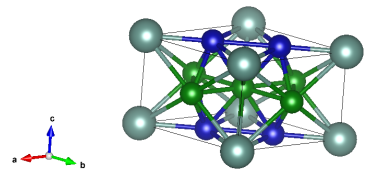

Fig: Crystal structure $\mathrm{YCO}_{5}$ (where gray balls are $\mathrm{Y}$, green balls are $\mathrm{Co}(3 \mathrm{~g})$ and blue balls are $\mathrm{Co}(2 \mathrm{c})$ ) 


\section{Density of States plots}

\section{Density of states plot of $\mathrm{YCo}_{5}$}

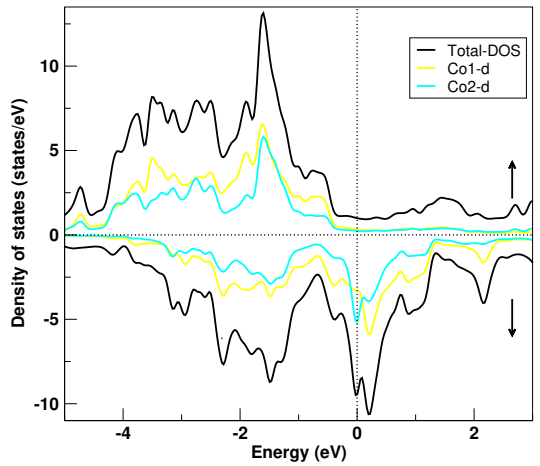

Fig : Density of states of parent compound $\mathrm{YCO} 5$
- Spin down $\rightarrow \mathrm{Co}(2 \mathrm{c})$ (major) with $\mathrm{Co}(3 \mathrm{~g})$

- Spin up $\rightarrow$ Co (3g) and a little from $\mathrm{Co}(2 \mathrm{c})$

- The magnetic moments obtained

\begin{tabular}{|c|c|c|}
\hline & GGA & GGA $+U$ \\
\hline $\mathrm{Y}(1 \mathrm{a})$ & $-0.20 \mu \mathrm{B}$ & $-0.25 \mu \mathrm{B}$ \\
\hline $\mathrm{Co}(2 \mathrm{c})$ & $1.57 \mu \mathrm{B}$ & $1.85 \mu \mathrm{B}$ \\
\hline $\mathrm{Co}(3 \mathrm{~g})$ & $1.60 \mu \mathrm{B}$ & $1.91 \mu \mathrm{B}$ \\
\hline Total & $7.20 \mu \mathrm{B}$ & $8.10 \mu \mathrm{B}$ \\
\hline
\end{tabular}

- Since Co on $\mathrm{YCO}_{5}$ is in intermediate spin state we have taken the value of on-site potential $U=3 \mathrm{eV}$ throughout this work 


\section{DOS and band plots}

Density of states of $\mathrm{Y}_{1-\mathrm{x}} \mathrm{Ca}_{\mathrm{x}} \mathrm{Co}_{5}$
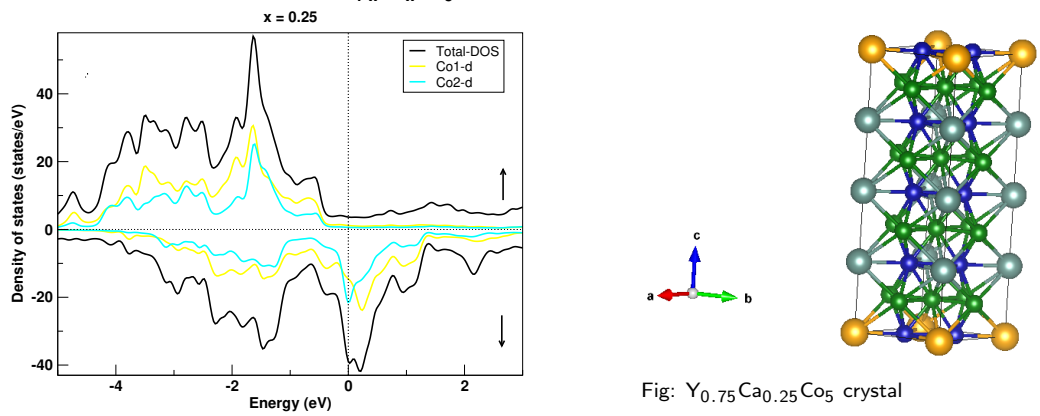

Fig: $\mathrm{Y}_{0.75} \mathrm{Ca}_{0.25} \mathrm{Co}_{5}$ crystal

Fig: Density of states of $\mathrm{Y}_{0.75} \mathrm{Ca}_{0 .}{ }_{25} \mathrm{Co}_{5}$

- Observed magnetic moment in $\mu \mathbf{B}$ of $Y_{0.75} \mathrm{Ca}_{0.25} \mathrm{Co}_{5}$

\begin{tabular}{|c|c|c|c|c|c|}
\hline & $\mathrm{Y}$ & $\mathrm{Ca}$ & $\mathrm{Co}(3 \mathrm{~g})$ & $\mathrm{Co}(2 \mathrm{c})$ & Total \\
\hline $\mathrm{GGA}$ & -0.20 & -0.10 & 1.71 & 1.58 & 31.08 \\
\hline$+U=3 \mathrm{eV}$ & -0.22 & -0.11 & 1.97 & 1.91 & 34.32 \\
\hline
\end{tabular}




\section{DOS and band plots}
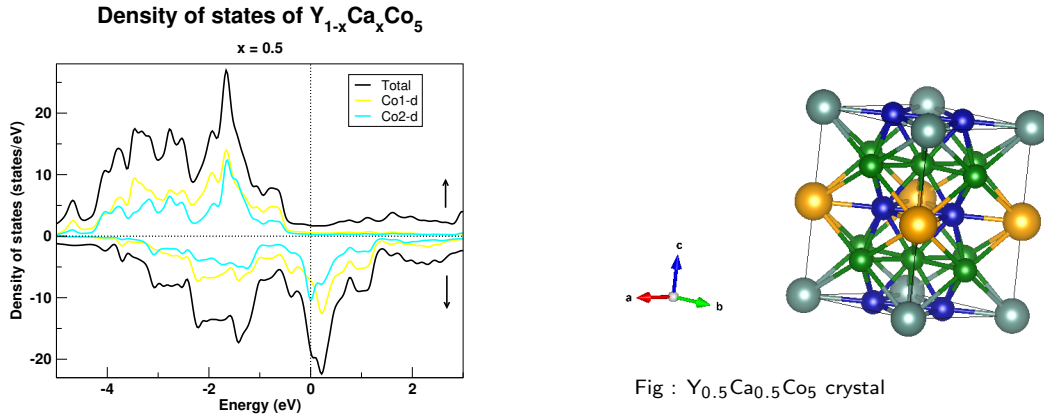

Fig : $\mathrm{Y}_{0.5} \mathrm{Ca}_{0.5} \mathrm{Co}_{5}$ crystal

Fig : Density of states of $\mathrm{Y}_{0.5} \mathrm{Ca}_{0.5} \mathrm{Co}_{5}$

- Observed magnetic moment in $\mu \mathrm{B}$ of $Y_{0.50} \mathrm{Ca}_{0.50} \mathrm{Co}_{5}$

\begin{tabular}{|c|c|c|c|c|c|}
\hline & $\mathrm{Y}$ & $\mathrm{Ca}$ & $\mathrm{Co}(3 \mathrm{~g})$ & $\mathrm{Co}(2 \mathrm{c})$ & Total \\
\hline $\mathrm{GGA}$ & -0.17 & -0.07 & 1.74 & 1.65 & 15.22 \\
\hline$+U=3 \mathrm{eV}$ & -0.13 & -0.06 & 1.96 & 1.91 & 17.22 \\
\hline
\end{tabular}




\section{DOS and band plots}

Density of states of $\mathrm{Y}_{1-\mathrm{x}} \mathrm{Ca}_{\mathrm{x}} \mathrm{Co}_{5}$
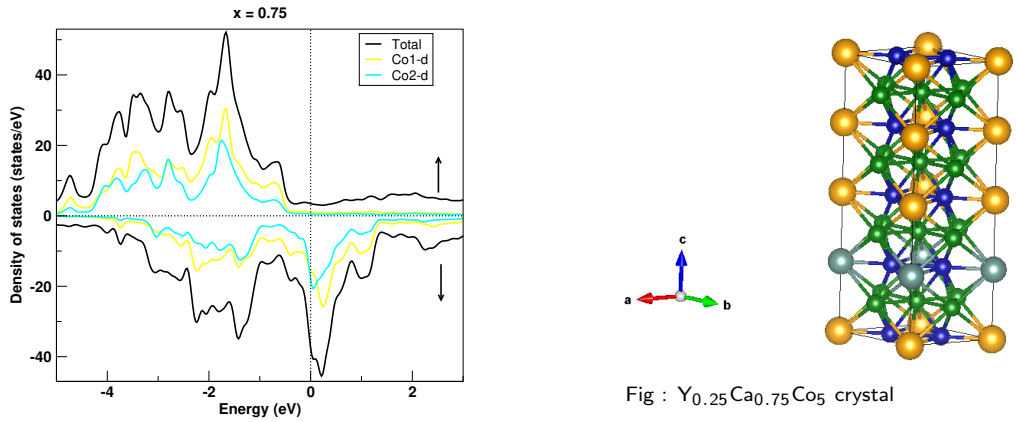

Fig : $\mathrm{Y}_{0.25} \mathrm{Ca}_{0.75} \mathrm{Co}_{5}$ crystal

Fig : Density of states of $\mathrm{Y}_{0.25} \mathrm{Ca}_{0.75} \mathrm{Co}_{5}$

- Observed magnetic moment in $\mu \mathrm{B}$ of $Y_{0.25} \mathrm{Ca}_{0.75} \mathrm{Co}_{5}$

\begin{tabular}{|c|c|c|c|c|c|}
\hline & $\mathrm{Y}$ & $\mathrm{Ca}$ & $\mathrm{Co}(3 \mathrm{~g})$ & $\mathrm{Co}(2 \mathrm{c})$ & Total \\
\hline $\mathrm{GGA}$ & -0.20 & -0.10 & 1.72 & 1.64 & 29.52 \\
\hline$+U=3 \mathrm{eV}$ & -0.17 & -0.07 & 1.98 & 1.93 & 35.71 \\
\hline
\end{tabular}




\section{Band plots}
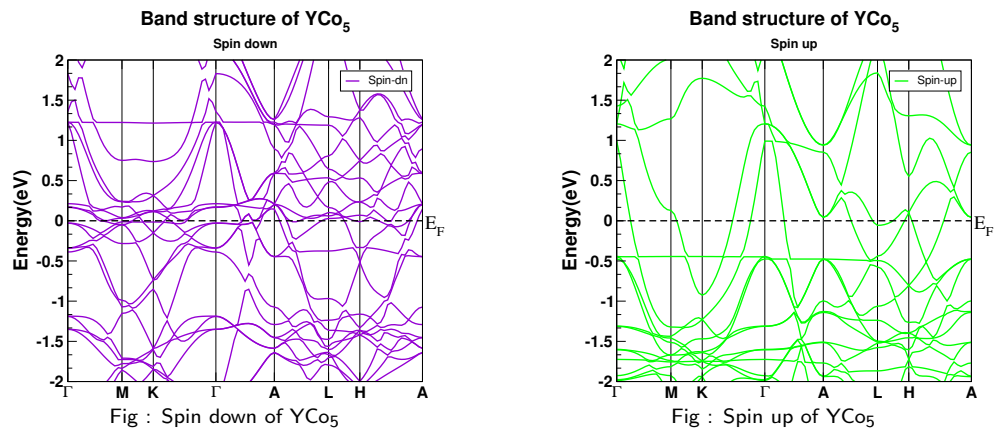

- Flat band is present in path $\Gamma-M-K-\Gamma-A$ in both spin-channels

- $\mathrm{E}_{F}=0.6095 \mathrm{eV}$ 


\section{Band plots}

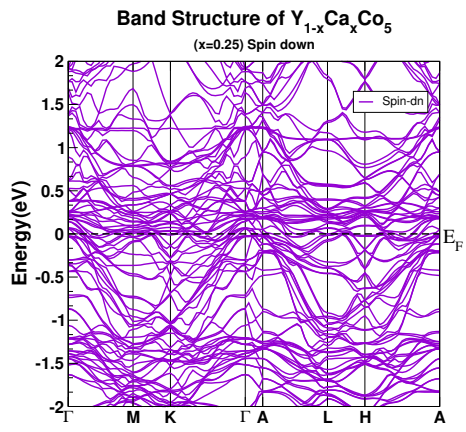

Fig : Spin down of $\mathrm{Y}_{0.75} \mathrm{Ca}_{0.25} \mathrm{Co}_{5}$
Band Structure of $\mathrm{Y}_{1-\mathrm{x}} \mathrm{Ca}_{\mathrm{x}} \mathrm{Co}_{5}$

$(x=0.25)$ Spin up

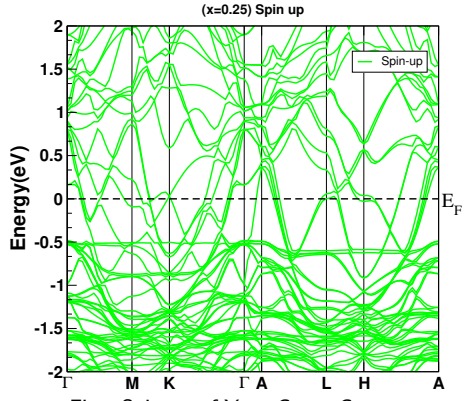

Fig : Spin up of $\mathrm{Y}_{0.75} \mathrm{Ca}_{0.25} \mathrm{Co}_{5}$

- Flat band is present in path $\Gamma-M-K-\Gamma-A$ in both spin-channels

- Another flat band is also seen in $A / L-L-H-H / A$

$\rightarrow \mathrm{E}_{F}=0.5464 \mathrm{eV}$ 


\section{Band plots}

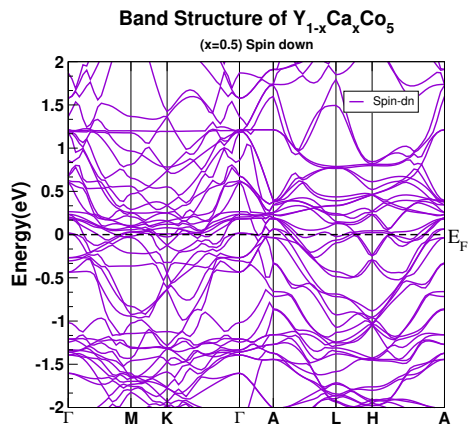

Fig : Spin down of $\mathrm{Y}_{0.5} \mathrm{Ca}_{0.5} \mathrm{Co}_{5}$

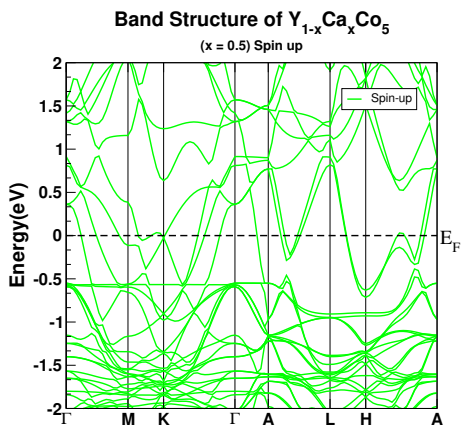

Fig : Spin up of $\mathrm{Y}_{0.5} \mathrm{Ca}_{0.5} \mathrm{Co}_{5}$

- Flat band is present in path $\Gamma-M-K-\Gamma-A$ in both spin-channels

- $\mathrm{E}_{F}=0.5476 \mathrm{eV}$ 


\section{Band plots}

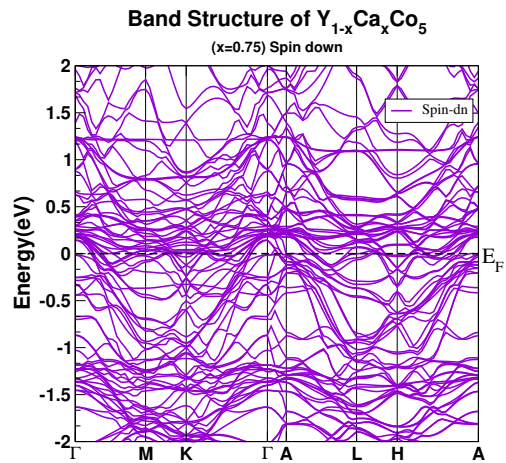

Fig : Spin down of $\mathrm{Y}_{0.25} \mathrm{Ca}_{0.75} \mathrm{Co}_{5}$

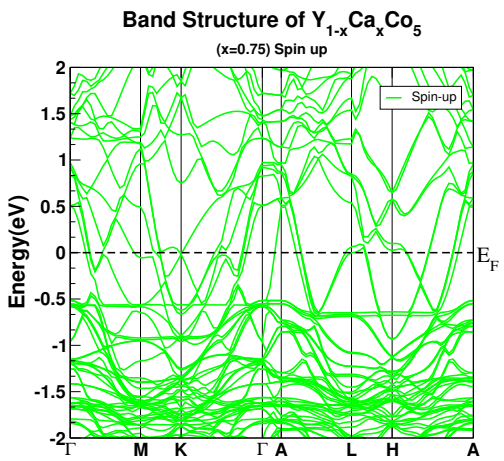

Fig: Spin up of $\mathrm{Y}_{0.25} \mathrm{Ca}_{0.75} \mathrm{Co}_{5}$

- Flat band is present in path $\Gamma-M-K-\Gamma-A$ in both spin-channels

- Another flat band is also seen in $A / L-L-H-H / A$

- $\mathrm{E}_{F}=0.6095 \mathrm{eV}$ 


\section{Conclusions}

- We investigated $Y_{1-x} C_{a x} C O_{5}$ with $(x=0,0.25,0.50$ and 0.75$)$ using DFT

- $Y_{1-x} C_{a} C_{0_{5}}$ for all values of $x$ are ferromagnets

- Magnetocrystalline Anisotropy Energy is found to decrease with increase in the concentration of dopant i.e $\mathrm{Ca}$

- The Fermi level shifted downwards with increase in the concentration of $\mathrm{Ca}$

- Flat band shifted away from Fermi level with increased doping for spin up and in case of spin down channel it shifted towards fermi level 


\section{Acknowledgments}

- Central Department of Physics, Tribhuvan University, Kathmandu, Nepal

- Condensed Matter Physics Research Center (CMPRC) Butwal, Rupandehi

- Ministry of Social Development, Gandaki Province, Nepal

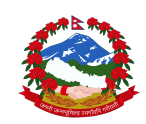

